\title{
Singlet-Triplet Dephasing in Asymmetric Quantum Dot Molecules
}

\author{
Ł. Marcinowski ${ }^{a}$, K. RoszAK ${ }^{a, b}$ And P. MAChnikOwski ${ }^{a, *}$ \\ ${ }^{a}$ Institute of Physics, Wrocław University of Technology \\ Wybrzeże Wyspiańskiego 27, 50-370 Wrocław, Poland \\ ${ }^{b}$ Department of Condensed Matter Physics, Faculty of Mathematics and Physics, Charles University \\ 12116 Prague, Czech Republic
}

We discuss pure dephasing of singlet-triplet superpositions in two-electron double quantum dots due to elastic phonon scattering. We generalize our previous results to a system built of two non-identical dots. We show that the asymmetry must be very strong in order to considerably affect the dephasing rate.

PACS numbers: 73.21.La, 03.65.Yz, 72.10.Di, 03.67.Lx

\section{Introduction}

Dephasing of singlet-triplet superpositions in two-electron quantum dot molecules (QDMs) is important for a possible implementation of quantum information processing in semiconductor systems [1]. In a recent work [2] we showed that elastic phonon scattering via virtual transitions to doubly occupied states, which is only possible in a singlet configuration, induces distinguishability of spin configurations and, therefore, leads to pure dephasing of spin superpositions. In Ref. [2] we studied a system of two identical dots. However, as quantum dots are artificial systems, one has to take into account unavoidable inhomogeneity of dot parameters when modeling the properties of the system. Therefore, in the present contribution, we generalize our previous result and study the phonon-induced dephasing process in an asymmetric QDM. We show that in the asymmetric QDM an additional dephasing channel appears, as compared to the symmetric one. Nonetheless, the dephasing rate is very weakly affected by the asymmetry unless the latter becomes very strong.

\section{Model}

The system under consideration is composed of two electrons in an asymmetric QDM built from two different gate-defined quantum dots $[3,4]$. The electrons are coupled to phonons by the usual charge-phonon interactions (deformation potential and piezoelectric couplings). We do not take any spin-environment interactions into account (neither direct, with nuclear spins, nor indirect, via spin-orbit coupling). The Hamiltonian of the system

* corresponding author; e-mail: pawel.machnikowski@pwr.wroc.pl is then

$$
H=H_{\mathrm{DQD}}+H_{\mathrm{ph}}+H_{\mathrm{int}} .
$$

The first term describes the electrons and has the form

$$
\begin{aligned}
& H_{\mathrm{DQD}}=\Delta \epsilon \sum_{s}\left(a_{\mathrm{L} s}^{\dagger} a_{\mathrm{L} s}-a_{\mathrm{R} s}^{\dagger} a_{\mathrm{R} s}\right) \\
& -t_{1} \sum_{s}\left(a_{\mathrm{L} s}^{\dagger} a_{\mathrm{R} s}+\text { H.c. }\right) \\
& +\frac{1}{2} \sum_{s, s^{\prime}} \sum_{i, j, k, l} V_{i j k l} a_{i s}^{\dagger} a_{j s^{\prime}}^{\dagger} a_{k s^{\prime}} a_{l s},
\end{aligned}
$$

where $a_{i s}, a_{i s}^{\dagger}$ are the electron annihilation and creation operators with $i=\mathrm{L}, \mathrm{R}$ denoting the left and right dot, respectively, and $s=\uparrow, \downarrow$ labeling the spin orientation. The first term in Eq. (1) accounts for the energy difference between single-electron states in the two dots. The second term represents single-particle inter-dot tunneling. The third term describes the Coulomb interaction, with $V_{i j k l}=V_{j i l k}=V_{k l i j}=V_{l k j i}$ (the wave functions may be chosen such that the matrix elements are real).

The Hamiltonian of the phonon reservoir is given by $H_{\mathrm{ph}}=\sum_{\boldsymbol{k}, \lambda} \hbar \omega_{\boldsymbol{k}, \lambda} b_{\boldsymbol{k}, \lambda}^{\dagger} b_{\boldsymbol{k}, \lambda}$, where $b_{\boldsymbol{k}, \lambda}, b_{\boldsymbol{k}, \lambda}^{\dagger}$ are annihilation and creation operators for a phonon from a branch $\lambda$ with a wave vector $\boldsymbol{k}$ and $\hbar \omega_{\boldsymbol{k}, \lambda}$ are the corresponding energies. The electron-phonon interaction is described by

$$
H_{\mathrm{int}}=\sum_{s, i} \sum_{\boldsymbol{k}, \lambda} F_{i}^{(\lambda)}(\boldsymbol{k}) a_{i s}^{\dagger} a_{i s}\left(b_{\boldsymbol{k}, \lambda}+b_{-\boldsymbol{k}, \lambda}^{\dagger}\right),
$$

where $F_{\mathrm{L} / \mathrm{R}}^{(\lambda)}(\boldsymbol{k})$ are coupling constants. We use the usual coupling constants for confined charges (see Refs. $[2,5]$ for explicit expressions).

The Hamiltonian $H_{\mathrm{DQD}}$ can be diagonalized in a simple way. The resulting singlet eigenstates can be written in the form 


$$
\begin{aligned}
& \left|S_{0}\right\rangle= \\
& \cos \frac{\phi}{2}\left[\cos \frac{\theta}{2}|(1,1) S\rangle-\sin \frac{\theta}{2}|(+) S\rangle\right]-\sin \frac{\phi}{2}|(-) S\rangle, \\
& \left|S_{1}\right\rangle= \\
& -\sin \frac{\phi}{2}\left[\cos \frac{\theta}{2}|(1,1) S\rangle-\sin \frac{\theta}{2}|(+) S\rangle\right]+\cos \frac{\phi}{2}|(-) S\rangle, \\
& \left|S_{+}\right\rangle=\sin \frac{\theta}{2}|(1,1) S\rangle+\cos \frac{\theta}{2}|(+) S\rangle,
\end{aligned}
$$

where $|( \pm) S\rangle=[|(2,0) S\rangle \pm|(0,2) S\rangle] / \sqrt{2}$ and $|(m, n) S\rangle$ denotes the configuration with $m$ electrons in the left dot and $n$ electrons in the right one. The corresponding energies are denoted by $E_{0}, E_{1}, E_{+}$.

Here $\theta$ describes mixing of the $|(1,1) S\rangle$ and $|(+) S\rangle$ configurations due to tunneling and Coulomb interactions. In the present work we fix $\theta=0.5$, which corresponds to $t_{1} /\left(E_{1}-E_{0}\right)=0.2$.

The angle $\phi$ accounts for state mixing due to system asymmetry. The latter may be due to a difference between the energies of doubly occupied configurations in the two dots or to asymmetry of off-diagonal Coulomb elements. Accordingly, we define two parameters

$$
\begin{aligned}
& \Delta=2 \Delta \epsilon+\frac{V_{\mathrm{RRRR}}-V_{\mathrm{LLLL}}}{2}, \\
& \eta=\frac{V_{\mathrm{RLRR}}+V_{\mathrm{LRRR}}-V_{\mathrm{LRLL}}-V_{\mathrm{RLLL}}}{2} .
\end{aligned}
$$

Neglecting the exchange terms compared to direct Coulomb energies, one finds $\sin \phi \approx[\sin (\theta / 2) \Delta-$ $\cos (\theta / 2) \eta] /\left(E_{1}-E_{0}\right)$.

The carrier-phonon coupling for the two-electron system is written in the eigenbasis of $H_{\mathrm{DQD}}$,

$$
H_{\mathrm{int}}=\sum_{\alpha, \beta, \boldsymbol{k}, \lambda} F_{\alpha \beta}^{(\lambda)}(\boldsymbol{k})|\alpha\rangle\langle\beta|\left(b_{\boldsymbol{k}, \lambda}+b_{-\boldsymbol{k}, \lambda}^{\dagger}\right),
$$

with $\alpha, \beta=S_{0}, S_{1}, S_{+}$. Then, the phonon spectral densities involving the low-energy state $\left|S_{0}\right\rangle$ are found,

$$
R_{\alpha \beta}(\omega)=\sum_{\boldsymbol{k}, \lambda} F_{S_{0} \alpha}^{(\lambda)}(\boldsymbol{k}) F_{S_{0} \beta}^{(\lambda) *}(\boldsymbol{k})|n(\omega)+1| \delta\left(|\omega|-\omega_{\boldsymbol{k}}\right),
$$

where $\alpha, \beta=S_{1}, S_{+}$and $n(\omega)$ is the Bose distribution. In contrast to the previously studied symmetric case, two channels of phonon scattering are now present, corresponding to the two different excited states $\left(S_{1}\right.$ and $\left.S_{+}\right)$ through which the phonons can scatter. The interference of different scattering paths is reflected by the presence of the whole family of spectral densities out of which only one, $R_{S_{1} S_{1}}(\omega)$ survives for $\phi \rightarrow 0$ and reduces to the spectral density found in Ref. [2].

Following the method worked out in our previous paper [2], we calculate the dephasing rate using the 4 th order time-convolutionless equation for the evolution of the density matrix describing the state of the two-electron system. From this, we extract the Markov limit, which yields the dephasing rate due to two-phonon (scattering) processes. Similarly as in the symmetric case [2], this process dominates over the one-phonon real transitions at low temperatures. The resulting dephasing rate is given by

$$
\begin{aligned}
\gamma & =\pi \mathcal{P} \sum_{\alpha, \beta} \int_{-\infty}^{\infty} \mathrm{d} \omega\left[\frac{R_{\alpha \beta}(\omega) R_{\alpha \beta}(-\omega)}{\left(\omega+\omega_{\alpha}\right)\left(\omega_{\beta}-\omega\right)}\right. \\
& \left.+\frac{R_{\alpha \beta}(\omega) R_{\alpha \beta}(-\omega)-R_{\alpha \beta}\left(\omega_{\alpha}\right) R_{\alpha \beta}\left(-\omega_{\beta}\right)}{\left(\omega-\omega_{\alpha}\right)\left(\omega-\omega_{\beta}\right)}\right],
\end{aligned}
$$

where $\omega_{\alpha}=\left(E_{\alpha}-E_{0}\right) / \hbar$ and $\mathcal{P}$ denotes the Cauchy principal value. The above equation generalizes the previously found formula to the asymmetric case.

In the calculations, we set the energy differences $E_{1}-$ $E_{0}=0.9 \mathrm{meV}, E_{+}-E_{0}=1.0 \mathrm{meV}$, the inter-dot distance $D=300 \mathrm{~nm}$, and use the material parameters as in Ref. [2].

\section{Results}

The pure dephasing rates resulting from the scattering process are shown in Fig. 1a,c as a function of $\Delta$ for a few values of $\eta$ and in Fig. 1b,d as a function of $\eta$ for a few values of $\Delta$. In all the cases, the dephasing time varies from about $1 \mu \mathrm{s}$ to several $\mu \mathrm{s}$. As long as the asymmetry is small, the dephasing rate is rather insensitive to it. Only for strong asymmetry, the dephasing rate grows considerably. One should keep in mind that the largest values of $\eta$ shown in Fig. 1 are rather unrealistic since this parameter describes the variation of exchange-like Coulomb terms which are themselves small. In a real structure, $\eta$ is likely to remain within a tenth of $\mathrm{meV}$. It is clear from Fig. 1 that in this parameter range the

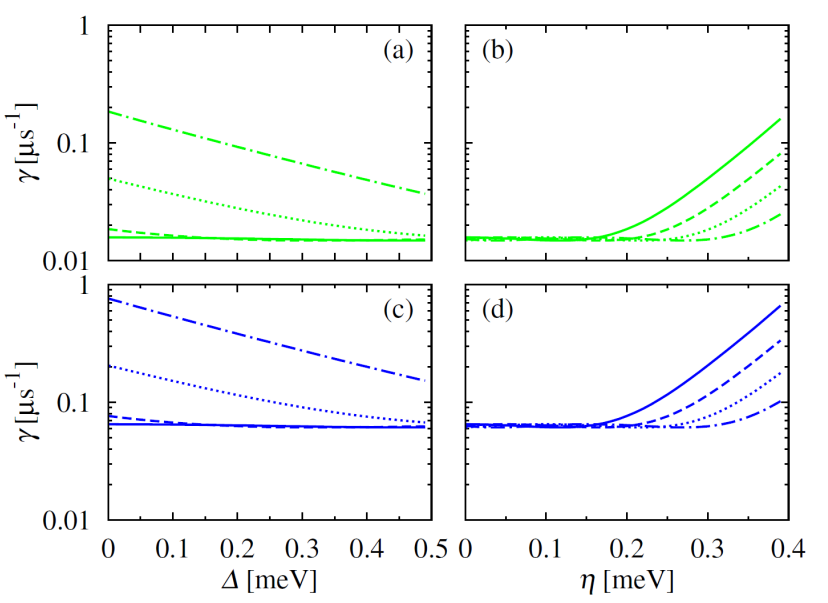

Fig. 1. Dephasing rate: (a),(c) as a function of $\Delta$ for $\eta=0$ (solid line), $0.2 \mathrm{meV}$ (dashed line), $0.3 \mathrm{meV}$ (dotted line), and $0.4 \mathrm{meV}$ (dash-dotted line); (b), (d) as a function of $\eta$ for $\Delta=0$ (solid line), $0.2 \mathrm{meV}$ (dashed line), $0.4 \mathrm{meV}$ (dotted line), and $0.6 \mathrm{meV}$ (dash-dotted line). In (a) and (b) $T=0.5 \mathrm{~K}$; in (c) and (d) $T=1 \mathrm{~K}$. 
dephasing rate is almost completely insensitive to $\eta$, as well as to $\Delta$.

\section{Acknowledgments}

This work was supported in part by the Czech Science Foundation (grant no. 202/07/J051).

\section{References}

[1] D. Loss, D.P. DiVincenzo, Phys. Rev. A 57, 120 (1998).
[2] K. Roszak, P. Machnikowski, Phys. Rev. B 80, 195315 (2009).

[3] J.R. Petta, A.C. Johnson, J.M. Taylor, E.A. Laird, A. Yacoby, M.D. Lukin, C.M. Marcus, M.P. Hanson, A.C. Gossard, Science 309, 2180 (2005).

[4] F.H.L. Koppens, J.A. Folk, J.M. Elzerman, R. Hanson, L.H. Willems van Beveren, I.T. Vink, H.P. Tranitz, W. Wegscheider, L.P. Kouwenhoven, L.M.K. Vandersypen, Science 309, 1346 (2005).

[5] A. Grodecka, P. Machnikowski, J. Förstner, Phys. Rev. B 78, 085302 (2008). 